\title{
Functional Smart Dispersed Liquid Crystals for Nano- and Biophotonic Applications: Nanoparticles-Assisted Optical Bioimaging
}

\author{
N. V. Kamanina, ${ }^{1,2}$ S. V. Likhomanova, ${ }^{1,3}$ Yu. A. Zubtcova, ${ }^{1}$ \\ A. A. Kamanin, ${ }^{4}$ and A. Pawlicka ${ }^{5}$ \\ ${ }^{1}$ Lab for Photophysics of Media with Nanoobjects, Vavilov State Optical Institute, Kadetskaya Liniya VO, Dom 5, \\ Korpus 2, St. Petersburg 199053, Russia \\ ${ }^{2}$ St. Petersburg Electrotechnical University (“LETI”), Professora Popova Street, Dom 5, St. Petersburg 197376, Russia \\ ${ }^{3}$ St. Petersburg University of Fine Mechanics and Optics, Sablinskaya Street 14, St. Petersburg 197101, Russia \\ ${ }^{4}$ Pavlov First St. Petersburg State Medical University, Lev Tolstoy Street 6/8, St. Petersburg 197022, Russia \\ ${ }^{5}$ IQSC-USP, Avenida Trabalhador São Carlense 400, 13566-590 São Carlos, SP, Brazil
}

Correspondence should be addressed to N. V. Kamanina; nvkamanina@mail.ru

Received 5 February 2016; Revised 15 April 2016; Accepted 20 April 2016

Academic Editor: Dan Wang

Copyright (C) 2016 N. V. Kamanina et al. This is an open access article distributed under the Creative Commons Attribution License, which permits unrestricted use, distribution, and reproduction in any medium, provided the original work is properly cited.

Functional nematic liquid crystal structures doped with nano- and bioobjects have been investigated. The self-assembling features and the photorefractive parameters of the structured liquid crystals have been comparatively studied via microscopy and laser techniques. Fullerene, quantum dots, carbon nanotubes, DNA, and erythrocytes have been considered as the effective nano- and biosensitizers of the LC mesophase. The holographic recording technique based on four-wave mixing of the laser beams has been used to investigate the laser-induced change of the refractive index in the nano- and bioobjects-doped liquid crystal cells. The special accent has been given to novel nanostructured relief with vertically aligned carbon nanotubes at the interface: solid substrate-liquid crystal mesophase. It has been shown that this nanostructured relief influences the orienting ability of the liquid crystal molecules with good advantage. As a result, it provokes the orientation of the DNA. The modified functional liquid crystal materials have been proposed as the perspective systems for both the photonics and biology as well as the medical applications.

\section{Introduction}

It is well known that the nematic liquid crystals (NLC) as the suitable model systems for the nanoparticles including have been regularly investigated and successfully considered by the different scientific and technical groups. The reason is connected with the fact that this process permits predicting and optimizing the best conditions to make innovative physical models describing the photorefractive and photoconductive effects as well as the dynamic one. Unique optical, nonlinearoptical, electro-, acoustic-, magneto-, and thermoproperties of LCs are widely applied to laser physics, optical information processing, display technology, microscopy, biology, and surface defects identification, as well as medicine. An important feature of LC molecules is a high orienting capacity, which can be used to create novel sensitive composite materials. LC orients particles suspended in them and acts as the model molecular matrix easily controlled by an applied external field. Particles become sensitive to operating external field. As a result, the orientation of the LC matrix changes too with good advantage. Thus, the mutual influence of the LC matrix and particles introduced in them have been testified. Moreover, the influence of the interface between LC and solid substrate on the materials volumetric characteristics has been also under the consideration.

At present time, a study of organics systems, specially dispersed liquid crystals, when properties of mesophase can be reinforced by doping of the novel dyes, fullerenes, carbon nanotubes, gold nanoparticles, quantum dots, shungites, grapheme oxides, and so forth, holds the great promise 
because they combine the properties of photosensitive components with their unique energetic levels and specific surface area and the electrooptical parameters of the LC mesophase too. Many types of the novel organic composites, the dispersed LC structures, and new nano- and biodopants have been realized and studied by complicated techniques [1-15].

In the present paper, the NLC systems structured with the 2-cyclooctylamino-5-nitropyridine (COANP)- $\mathrm{C}_{70}$ complex, quantum dots (QDs), DNA, and so forth have been comparatively studied as effective media for checking the laserinduced change of the refractive index and quasismectic selfassembly features and to control the effect of the interface with carbon nanotubes (CNTs) on the volumetric LC parameters, for example, on the spectral parameters and on the refractive index too. To improve the refractive characteristics and change the order parameters of this structure, the role of the intermolecular charge transfer complex between the donor fragment of organic molecules and effective intermolecular electron acceptor has been revealed and confirmed. The results obtained have been compared with those typical for the pure LC cells. Moreover, the orientation and the visualization of the DNA in the NLC mesophase with novel interface relief have been shown. The extended area of the application, including the medicine area, has been predicted.

\section{Materials and Experimental Conditions}

Nematic liquid crystal (NLC) from the inertial classical cyanobiphenyl group has been chosen as the flexible matrix. Let us remember that, from one side, the NLC media permit orienting the nano- and bioparticles included in that with good advantage, and, from other side, the properties of the NLC can be easy modified via intermolecular doping. LC cells have been assembled in $S$-configuration with a gap width of 7-10 micrometers. LC films have been placed onto glass substrates covered with transparent conducting layers based on ITO contacts. The transparent conducting ITO has been modified via CNTs laser deposition technique with additional treatment with surface electromagnetic wave (SEW). The general view of the LC structure is shown in Figure 1. The arrow on the glass shows the direction of processing by SEWs. It should be remarked that the dimension of relief grooves shown in Figure 1(b) is connected with the wavelength of the $\mathrm{CO}_{2}$ laser of 10.6 micrometers.

2-Cyclooctylamino-5-nitropyridine (COANP) conjugated monomer that was studied carefully before in [16-20] has been used as a donor molecule to create the intermolecular charge transfer complex (CTC) with the fullerenes, CNTs, QDs, DNA, and so forth. It should be mentioned that the COANP system is a good model with an effective initial intramolecular charge transfer process between $\mathrm{NH}$-donor group and $\mathrm{NO}_{2}$-acceptor. This intramolecular interaction can be easily transferred to intermolecular one by modification via the nanoobjects sensitization $[21,22]$. The intermolecular CTC has been formed in this $\pi$-conjugated organic system doped with fullerene $\mathrm{C}_{60}$ and $\mathrm{C}_{70}$, QDs, and so forth due to the large electron affinity energy of fullerene (it is close to $2.65 \mathrm{eV}$ ) or QDs (this value is placed in the range of $3.8-4.2 \mathrm{eV}$ ) that drastically exceeds the electron affinity energy of an intramolecular acceptor fragment of COANP because last value is close only to $0.45 \mathrm{eV}$. The destructive and nondestructive control testing of the formation of the intermolecular CTC, namely, $\mathrm{C}_{70}-\mathrm{NO}_{2}$, has been supported by results of the nonlinear absorption study [23, 24], Z-scanning technique [25, 26], IRspectral shift observation, mass-spectral and photorefractive experiments [22, 24, 26], order parameters changing [27], DSC analysis [28], quantum-chemical simulations [29], and so forth. For example, via DSC analysis, it has been shown that all transition temperature values have been shifted (such as a melting point, a point of crystallization, and a glass transition temperature) in the case of adding some amount of fullerene $\mathrm{C}_{70}$ in COANP. Moreover, recently, it has been shown by us that the same liquid crystal matrix photorefractive parameters have been changed not only via nanosensitization but via bioobjects doping too [30]. The intermolecular CTC have been supported by IR-spectral shift and order parameters increase as well.

The laser-induced change of the refractive index has been studied at the wave length of $532 \mathrm{~nm}$ under the RamanNath diffraction conditions [34-36]. Raman-Nath diffraction condition is realized in the case when the recorded grating period is larger than the thickness $d$ of the treated sample. Beam energies incident on and transmitted through the sample in the first-order diffraction can be measured. Our experiments have been made in the nanosecond pulsed regime at the spatial frequency of $90-130 \mathrm{~mm}^{-1}$ and at the laser energy density ranged from 0.01 to $0.6 \mathrm{~J} \cdot \mathrm{cm}^{-2}$. The technical experimental scheme has been analogous to that explained before in detail in $[37,38]$ and recently shown in [30] in the modified variant.

\section{Results and Discussion}

Let us briefly consider, for example, the effect of the nanostructuration of the LC media via the intermolecular CTC. We should consider a small local volume of the matrix model medium substantially smaller than the incident wavelength of the laser beams used to create the thin holographic grating. It is well known that, for a system with the dimensions smaller than the optical operating wavelength $(\lambda$ is $532 \mathrm{~nm}$ in our experiment; for comparison, LC molecules length is 15-100 angstroms, and fullerene molecules are $0.65-0.7 \mathrm{~nm}$ in size), the most important optical characteristic is the induced dipole, whose dependence on the applied local field can be expressed through dipole polarizabilities $\alpha^{(n)}$. These are, in their turn, related to the nonlinear susceptibility $\chi^{(n)}$ and are inversely proportional to the considered unit cell volume $v$. It has been shown in $[24,39,40]$ that thirdorder nonlinear susceptibility $\chi^{(3)}$, which is responsible for the change in the local volume polarizability in COANP fullerene-based LC systems, is much larger than that one for the pure nonsensitized structures. This value (namely, cubic nonlinearity) can be evaluated for these materials via the four-wave mixing technique from the photoinduced change of the refractive index. The gained polarization provides 


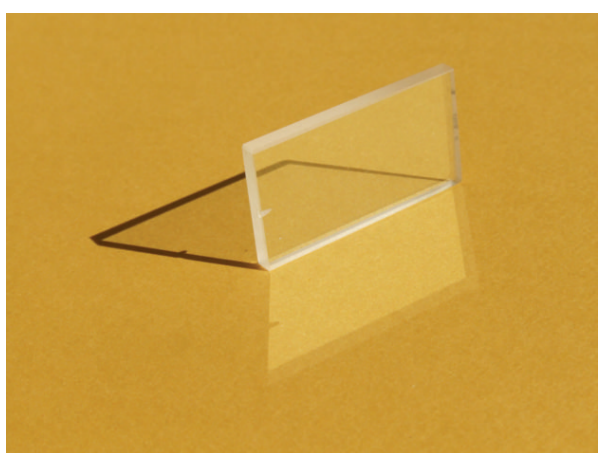

(a)

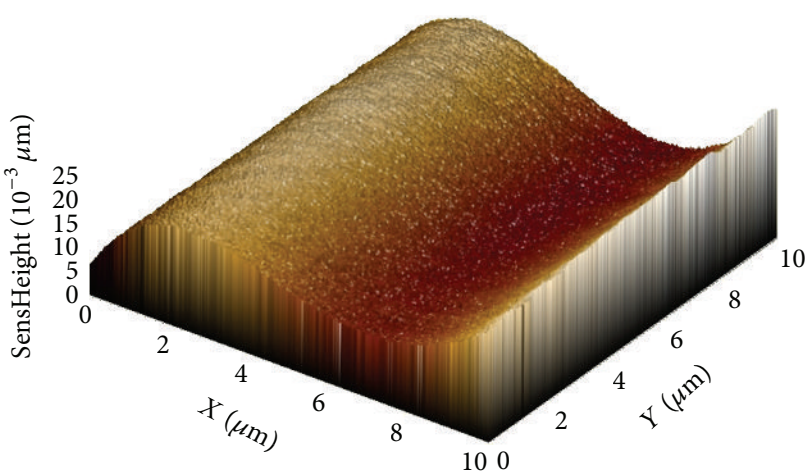

(b)

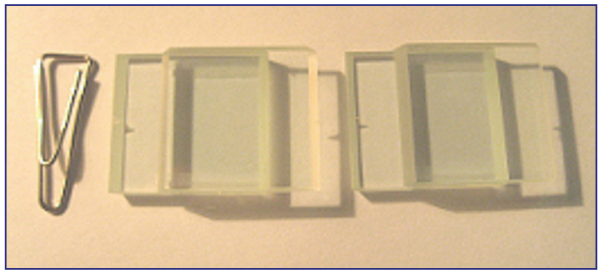

(c)

FIGURE 1: Glass substrate with ITO conducting coatings and laser-deposited carbon nanotubes additionally treated with SEW ((a) photo of the substrate), ITO+CNTs relief+SEW ((b) ASM image), and general view of the nano- or biostructured LC cells ((c) photo of the real cell with the dimension of $20 \times 22 \mathrm{~mm}$ ).

TABLE 1: Laser-induced change of the refractive index $\Delta n_{i}$ in the structured LC cells treated at $\lambda=532 \mathrm{~nm}$.

\begin{tabular}{|c|c|c|c|c|c|c|}
\hline Structure & $\begin{array}{c}\text { Dopants } \\
\text { content, wt.\% }\end{array}$ & $\begin{array}{l}\text { Energy density, } \\
\mathrm{J} \cdot \mathrm{cm}^{-2}\end{array}$ & $\begin{array}{l}\text { Spatial } \\
\text { frequency, } \\
\mathrm{mm}^{-1}\end{array}$ & $\begin{array}{l}\text { Pulse duration, } \\
\text { ns }\end{array}$ & $\Delta n_{i}$ & Ref. \\
\hline Pure LC & 0 & \multicolumn{3}{|c|}{$0.2 \mathrm{~W} \cdot \mathrm{cm}^{-2}$ at $514.5 \mathrm{~nm}$} & $0.16 \times 10^{-3}$ & {$[31]$} \\
\hline LC based on COANP- $\mathrm{C}_{70}$ & 5 & $17.5 \times 10^{-3}$ & 100 & 20 & $1.4 \times 10^{-3}$ & {$[24]$} \\
\hline LC based on COANP-C & 1 & $30 \times 10^{-3}$ & 90 & 10 & $1.45 \times 10^{-3}$ & Now \\
\hline LC based on COANP-C $\mathrm{C}_{70}$ & 1 & $30 \times 10^{-3}$ & 130 & 10 & $1.1 \times 10^{-3}$ & Now \\
\hline LC based on COANP-CNTs & 0.5 & $18.0 \times 10^{-3}$ & $90-100$ & $10-20$ & $3.2 \times 10^{-3}$ & [32] \\
\hline LC based on COANP- $\mathrm{C}_{70}$ & 0.5 & $30 \times 10^{-3}$ & 100 & 10 & $1.2 \times 10^{-3}$ & [33] \\
\hline LC based on COANP-CNTs & 0.1 & $30 \times 10^{-3}$ & 100 & 10 & $2.8 \times 10^{-3}$ & [33] \\
\hline LC based on DNA* & 0.1 & 0.1 & $90-120$ & & $1.39 \times 10^{-3}$ & {$[30]$} \\
\hline $\begin{array}{l}\text { LC based on QDs } \\
\text { CdSe }(\mathrm{ZnS})+\mathrm{DNA}\end{array}$ & 0.1 & 0.1 & $90-120$ & & $1.35 \times 10^{-3}$ & {$[30]$} \\
\hline $\begin{array}{l}\text { LC based on QDs } \\
\text { CdSe(ZnS)+DNA }\end{array}$ & 0.1 & 0.1 & 130 & & $1.0 \times 10^{-3}$ & Now \\
\hline
\end{tabular}

${ }^{*}$ Content of DNA (salmon fish) in the water solution was $\sim 4.72 \mathrm{~g} \cdot \mathrm{L}^{-1}$, and the relation between LC and DNA was $5: 1$.

a strong interaction between new domains with activation of the intermolecular CTC. This mechanism provokes the self-assembly features and increases of the refractivity and conductivity when NLC has been doped with nano- or bioobjects.

The basic photorefractive results have been shown in Table 1 including the comparative data obtained in the previously made studies; see [24, 30-33].

For better understanding, the data are presented in Table 1; the nature of the included particles, variation of their concentration, value of the energy density, and range of the spatial frequency are shown. The induced birefringence $\Delta n_{i}$ clearly demonstrates the creation of the CTC via possible changes in the charge transfer electron pathway and the amount of charge. It will lead to changes in the electric field gradient, dipole moment (proportional to the product of charge and distance), and mobility of charge carriers. This process permits proposing the method to make the LC systems with the increased activated birefringence.

In addition, the barrier-free charge transfer will be influenced by competition between the diffusion and drift of carriers during the creation of diffraction patterns with 


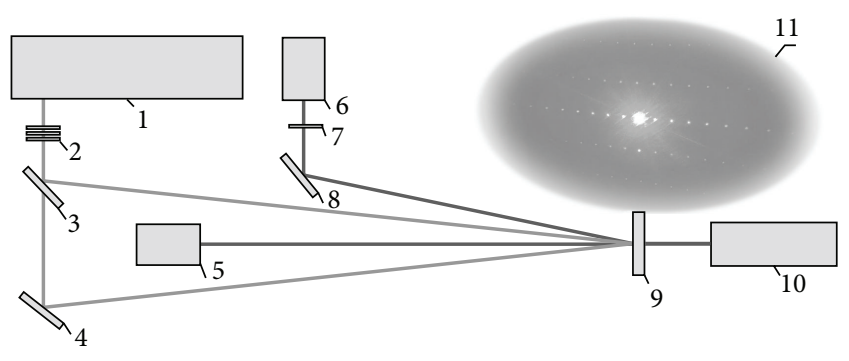

FIGURE 2: General view of the scheme and of the recorded grating on the organics under the Raman-Nath diffraction condition. 1, Ndlaser with the converter to 2nd harmonic; 2 and 7, filters; 3, 4, and 8 , mirrors; 5 and 6 , photodetectors; 9 , samples; 10 , an additional continuous $\mathrm{Nd}$ - or He-Ne-laser to readout; 11, view of the grating.

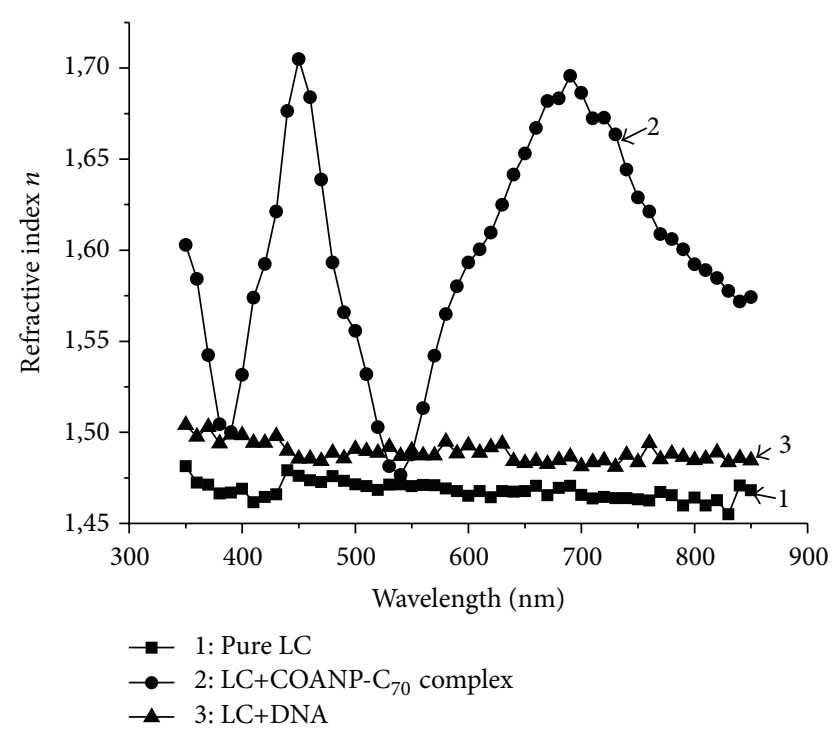

FIGURE 3: Spectral dependence of the refractive index $n$ via the nanoand biostructuration of the NLC mesophase.

various periods and, hence, differing charge localization at the grating nodes and antinodes. Indeed, in the case of a nanocomposites irradiated at small spatial frequencies (large periods of recorded grating), a drift mechanism of the carrier spreading in the electric field of an intense radiation field will most probably predominate, while, at large spatial frequencies (short periods of recorded grating), the dominating process is diffusion. The general view of the experimental holographic scheme and the grating recorded on the studied media is shown in Figure 2. It should be noticed that so many orders of the diffraction can be observed which corresponded not to the Bragg diffraction regime but, namely, to the Raman-Nath one. It should be mentioned that the classical ellipsometry method has been used as an additional one in order to support the increase of the refractive parameters. Really, the data shown in Figure 3 support this fact. The experiments have been performed using Horiba Jobin Yvon Uvisel device with the PSA-scheme.

One can see from Figure 3 that an introduction of the nano- and bioparticles provokes the increase of the refractive index in comparison with the same value in regard to pure LC. In the current experiments, the intermolecular CTC based on the COANP- $\mathrm{C}_{70}$ has been used and the DNA molecules have been applied. The nano- and biostructuration process leads to activate the self-assembly mechanism and permits observing the new large oriented numerous domains in the doped LC media. Finally, the structured LC composites with the new domains that have other orientation and order parameters have been testified before for the pure nematic LC media.

The new domains activated in the LC via intermolecular CTC formation are shown in Figure 4. One can observe the pictures with $2 \mathrm{D}$ or $3 \mathrm{D}$ local area which indicate the quasismectic transition from inertial nematic pure LC mesophase to polarized structured LC composites. The same effect has been shown in the previously reported paper [27] with checking the change of the transition temperature from the nematic phase (61 degrees) to quasismectic one (48 degrees) and via increase of the order parameters supported by NMR-analysis too. For comparison, the pure LC, LC+COANP- $\mathrm{C}_{70}$ CTC, LC with DNA, and LC with DNA and QDs are shown. The scale under Figures 4(a) and 4(b) is equal to 10 microns. The scale for Figures 4(c) and 4(d) is equal to 500 microns. One can see the appearance of the domains under the nano- and biostructuring.

It should be mentioned that the use of the luminescent QDs permits visualizing the DNA molecules in the LC media at the relief with CNTs. The basic views of the novel relief which has been used for the identification of the DNA and testing its form are shown in Figure 5. Different optical methods based on the optical spectroscopy and based on the AFM-analysis have been applied in order to support these results. Moreover, it should be noticed that both approaches indicate the coinciding profile of the made relief with the wavelength of the IR-laser operated at the wavelength of 10.6 micrometers.

It should be noticed that CNTs structured relief made on the ITO-coatings has been developed in vacuum using $p$ polarized radiation of the IR quasi-CW $\mathrm{CO}_{2}$ laser operating at the wavelength of $10.6 \mu \mathrm{m}$ and at the power of $30 \mathrm{~W}$. As an addition, relief has been modified by a surface electromagnetic wave (SEW) in order to have the orienting LC ability with good advantage. The detailed 2D and 3D AFM-images of the relief are shown in Figure 6.

During the deposition, the CNTs were oriented in the vertical position by applying the electric field with the strength of $100-600 \mathrm{~V} / \mathrm{cm}$. This procedure permits obtaining the significantly modified ITO-coatings characteristics due to the fact that CNTs have been covalent bonded with the ITO-layers that influences the transmission spectrum and the mechanical and laser strength of the material surface, as well as decreases the roughness [41-43]. For example, the application of CNTs leads to the formation of an effective layer at the substrate-CNT interface, since the nanotubes (with a refractive index of $n=1.1$ ) significantly reduce the Fresnel reflection losses by a factor of about 20. To support the change of the refractive interface parameters, the ellipsometry method has been used once again. Figure 7 presents the data in regard to decrease of the refractive index 


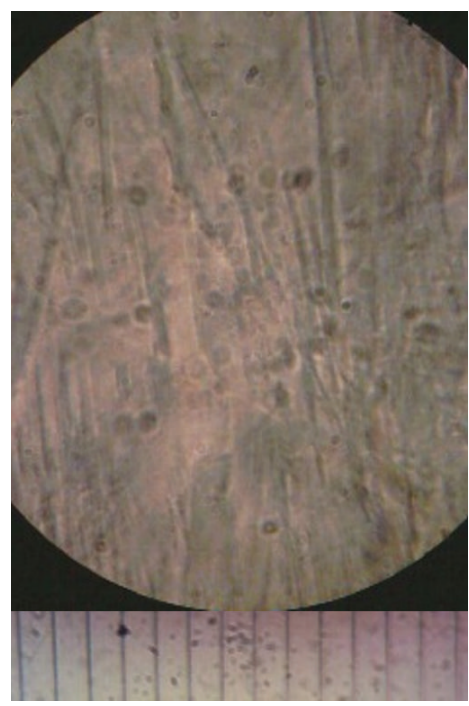

(a)

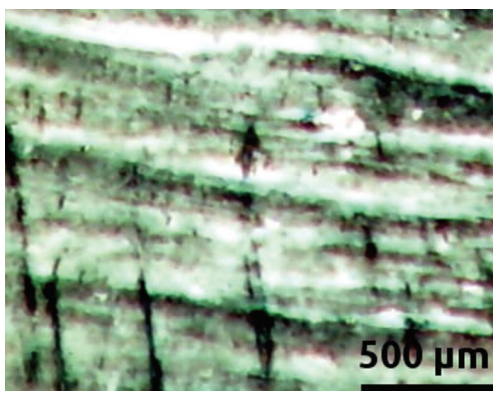

(c)

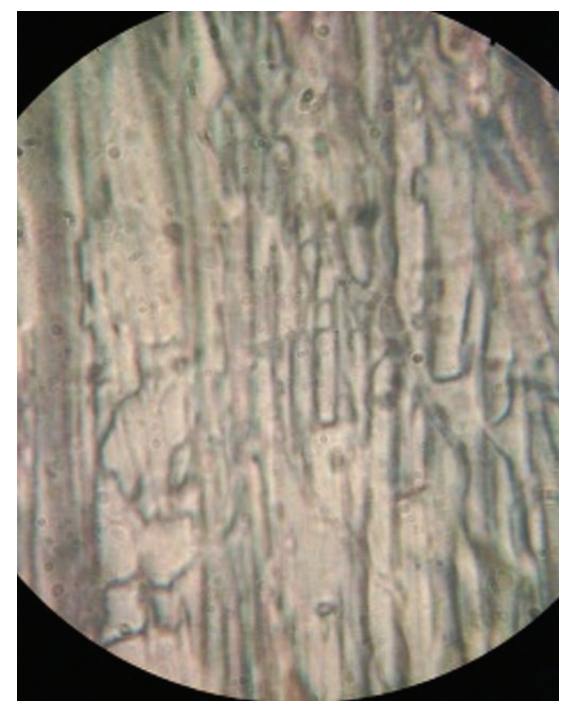

(b)

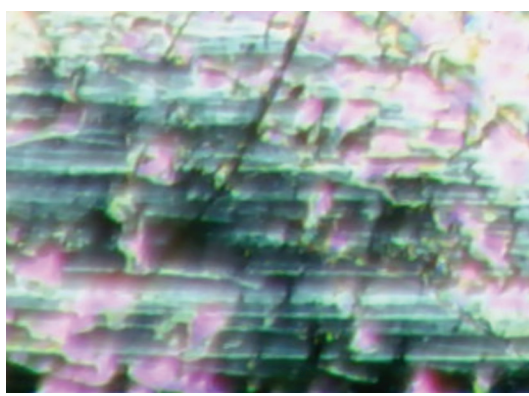

(d)

Figure 4: Pure NLC (a); NLC+COANP-C ${ }_{70}$ CTC (b); NLC structured with DNA (c); and NLC based on QDs CdSe(ZnS)+DNA (d).

of the ITO-coatings when CNTs have been laser deposited at the interface and oriented via applying the electric field with the strength of $100 \mathrm{~V} / \mathrm{cm}$.

The data presented in Figure 7 are correlated with the fact that transparency of the ITO-layer under the conditions with different treatment methods can be changed dramatically. This fact is shown in Figure 8. CNTs have been oriented with different electric field placed in the range from 100 to $600 \mathrm{~V} / \mathrm{cm}$. It permits obtaining the promising LC cell with this optimizes relief in order to improve the visualization and orientation of the CTC based on the nano- and bioobjects doping.

It should be drawn to attention that the relief mentioned above has been nontoxic and can provoke the orientation of the DNA molecules successfully. This relief can be proposed to orient other bioobjects, for example, erythrocytes, stomatocyte, and poikilocytes, in order to check their form and configuration as the express approach.

Let us remember that we have the practice to orient the bioobjects in the LC media. Really, the orientation of other bioobjects, such as the erythrocytes, has been made by us and reported early in [44-46]. The results of these studies showed that the structurization of an NLC mesophase can be induced not only by the introduction of dyes or fullerene-containing CTC complexes but also by adding of the nonelectrically neutral biological components such as human blood erythrocytes. Good prospect has been opened by the investigations in this direction using the methods of dynamic holography for the observation of diffraction on the ordered biaxial structures and the NMR for the monitoring of changes in the NLC order parameter in the presence of erythrocytes. These investigations have been also useful for medicine by providing the means of monitoring changes in the shape of human blood cells using NLC mesophase.

Thus, in the current study, the same affect has been revealed and supported for the DNA by applying the different techniques. It has provoked the modified improved properties of the LC systems by both the nano- and the bioobjects structuration of the body of the LC and by the nanostructuration of the interface. These evidences are supported via four-wave mixing technique and via selfassembling microscopy observation as well as via some innovative theoretical idea about the dipole increase in the polarized composites, which have been explained in detail in the previously published papers [38-40]. Moreover, some relief modification influences on the refractive and spectral 


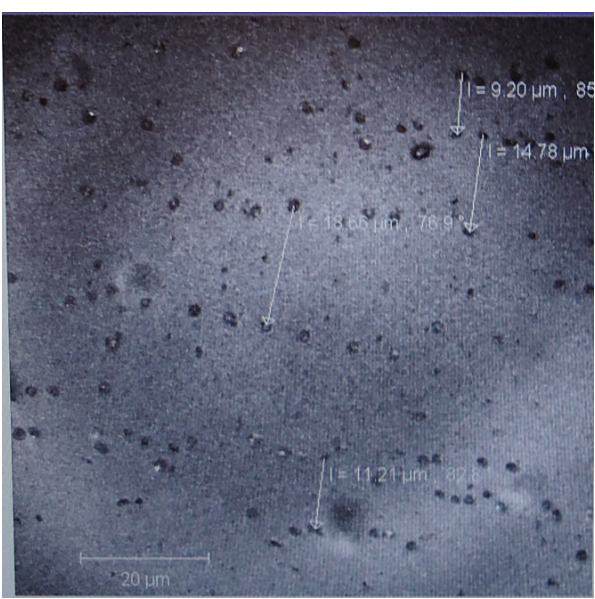

(a)

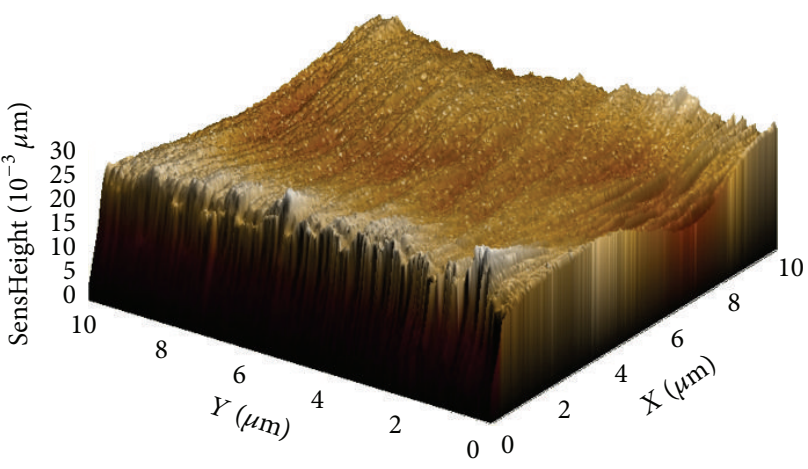

(b)

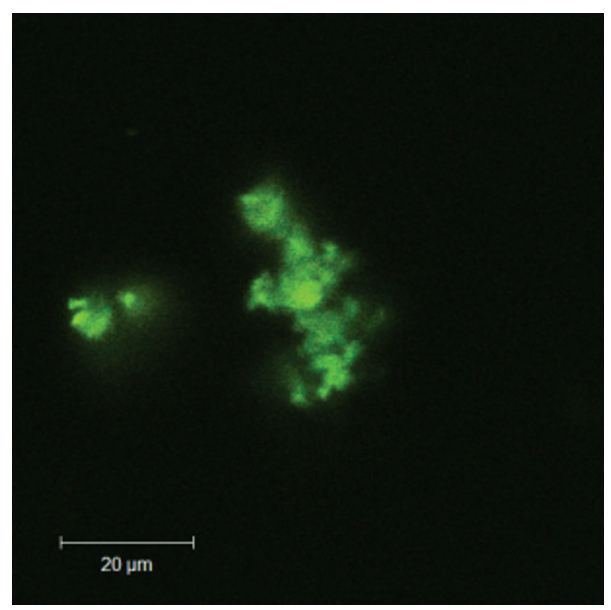

(c)

FIgURE 5: CNTs structured relief made on the ITO-coatings with the period of $\sim 10$ micrometers after IR-laser treatment ((a) optical microscopy studied and (b) AFM studied) and DNA molecule visualized at this relief (c) when LC based on the QDs CdSe(ZnS)+DNA has been investigated.

properties of the LC mesophase are shown now and previously have been presented in [47] when LCs have been doped with the different types of the lanthanides nanoparticles.

\section{Conclusions}

To summarize the results, one can testify the following basic conclusions:

(i) Nano- and biostructuration of the nematic LCmesophase body provokes the efficient self-assembly effect that connects with the increase of the order parameters and reveals the $2 \mathrm{D}$ and $3 \mathrm{D}$ LC systems instead of the $1 \mathrm{D}$ ones.

(ii) Nano- and biostructuration of the nematic LCmesophase body leads to increase of the birefringence that can be checked via holographic recording technique. (iii) Nanostructuration of the interface between LC mesophase and solid substrate directly influences on the decrease of the refractive index of the ITOcoatings due to the effective covalent bonding of the CNTs with the smaller refractive parameters.

(iv) Additional treatment of the nanostructured interface between LC mesophase and solid substrate with surface electromagnetic wave provokes the development of the novel orienting nontoxic relief responsible for the promising orientation of the DNA molecules, which can be visualize at these conditions with good advantage.

(v) Structuration processes mentioned above of the LC body and of the interface between LC mesophase and solid substrate extend drastically the area of the application of the nematic LC not only for the technical optoelectronics and theoretical nanophotonic applications but for the biology and medical use 


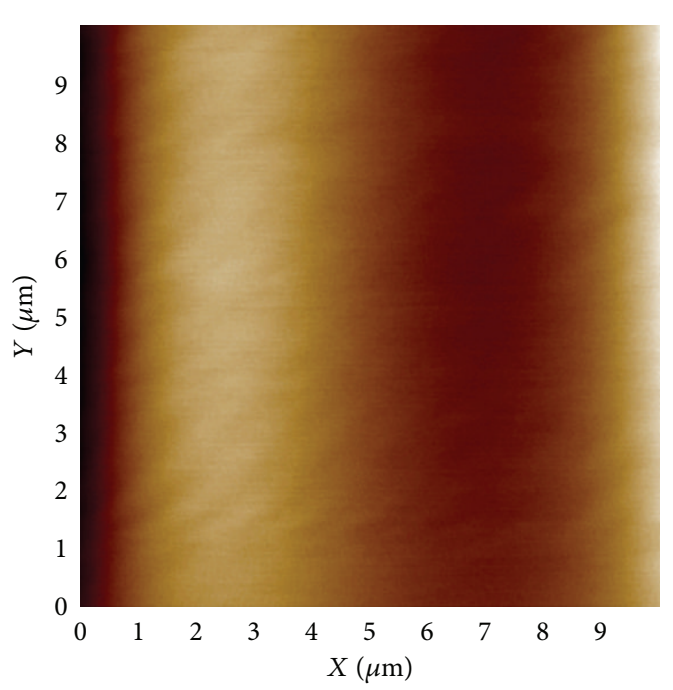

(a)

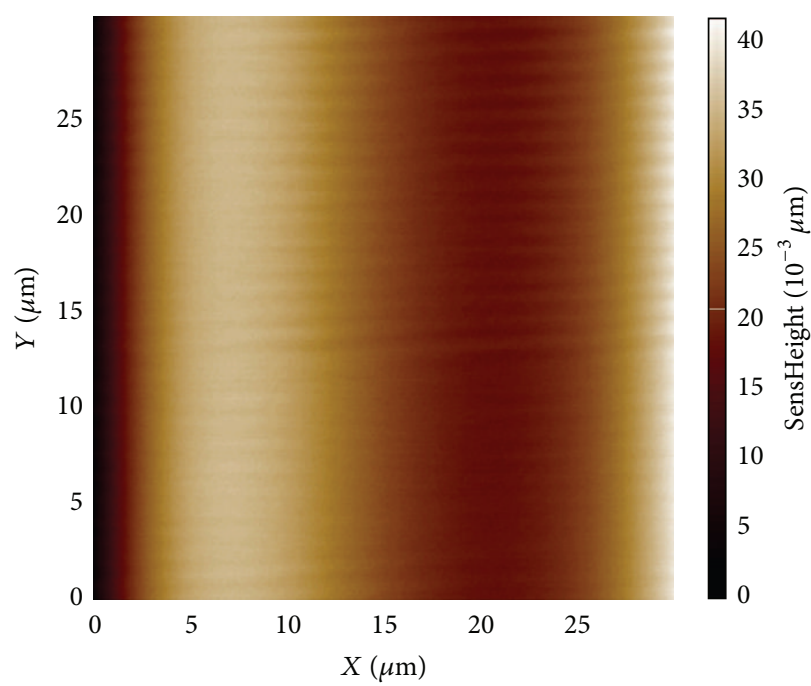

(c)

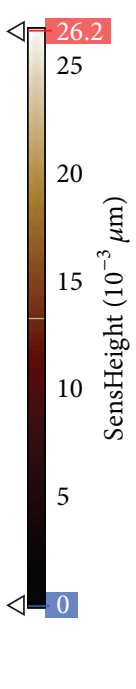

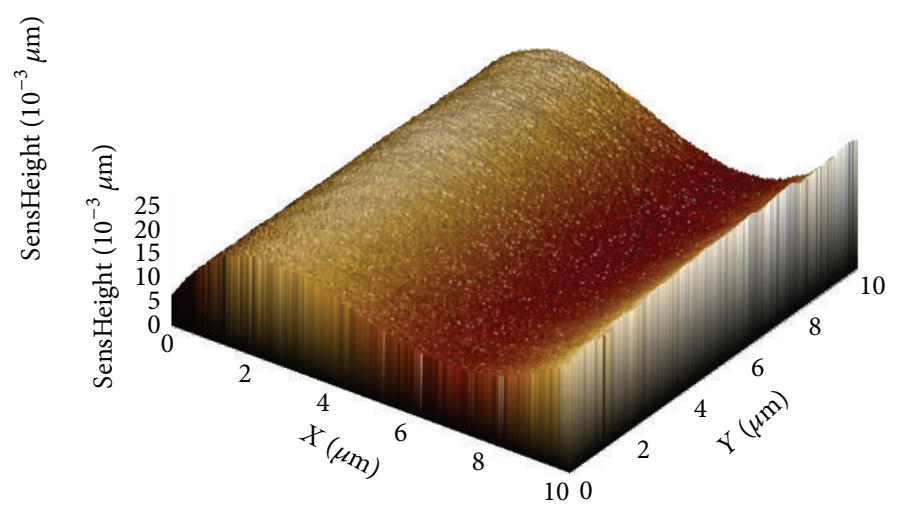

(b)

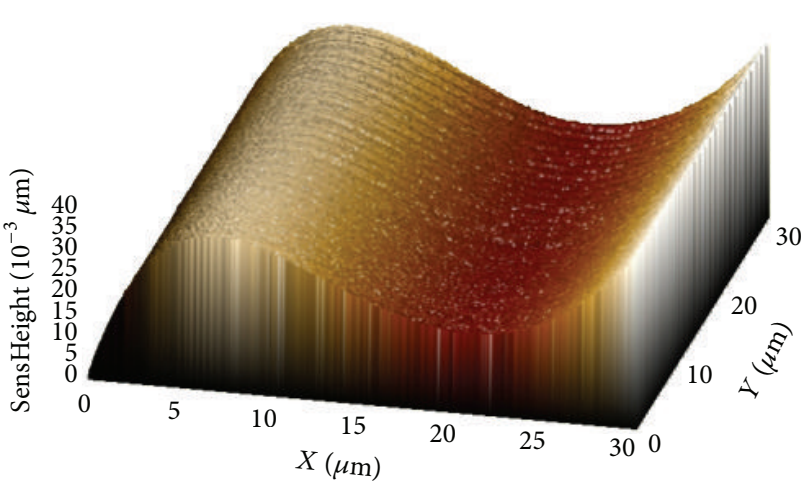

(d)

FIGURE 6: Different view of the relief developed: (a) ITO+CNTs+SEW 10 microns 2D-image; (b) ITO+CNTs+SEW 10 microns 3D-image; (c) ITO+CNTs+SEW 30 microns 2D-image; (d) ITO+CNTs+SEW 30 microns 3D-image.

too due to express analysis use to test the form or configuration of the bioobjects.

Indeed, it should be remarked that, for future experimental evidences of the intermolecular charge transfer complex formation in the nematic LC doped with both the nanoobjects and the bioobjects, the scanning electron microscopy supporting should be used.

\section{Disclosure}

Some parts of the results presented in the current paper have been discussed at the scientific seminar, Czestochowa University (December 12, 2012, Poland), at the Nanotechnology Session in the framework of the MMT-2014 Conference (July 28-August 1, 2014, Ariel, Israel), at and the 3rd International
Workshop on Nano and Bio-Photonics (6-11 December 2015, Cabourg, France).

\section{Competing Interests}

The authors declare that they have no competing interests.

\section{Acknowledgments}

The authors would like to acknowledge their colleagues: Professor D. P. Uskokovic (Institute of Technical Sciences of the Serbian Academy of Sciences and Arts, Belgrade, Serbia); Brazilian Ph.D. student Rodrigo Sabadini (from A. Pawlicka Lab., Brasil); Dr. Guillaume Micouin (from Ch. Andraud Lab., France), Professor Ch. Andraud, and Professor F. Kajzar (Laboratoire de Chimie, UMR CNRS 5182, Ecole Normale 


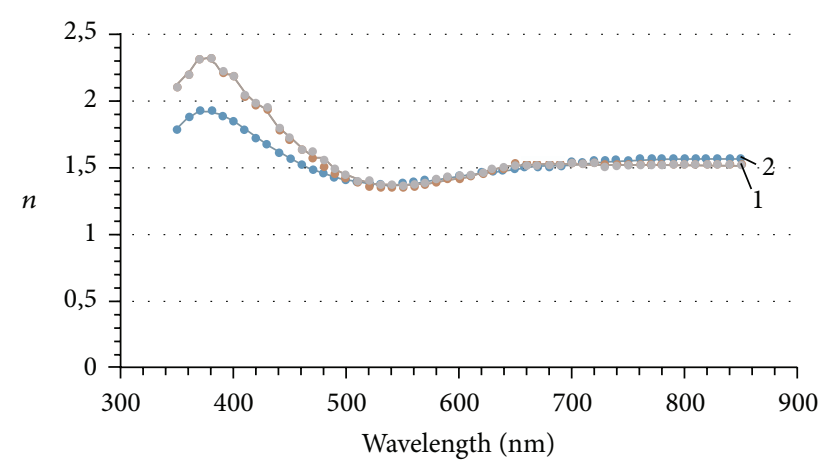

1: ITO pure

2: ITO+CNT100

FIGURE 7: Dependence of the refractive index on wavelength of the pure ITO-coatings at the glass substrate and of the ITO-layer structured with CNTs deposited under the condition when electric field has been used.

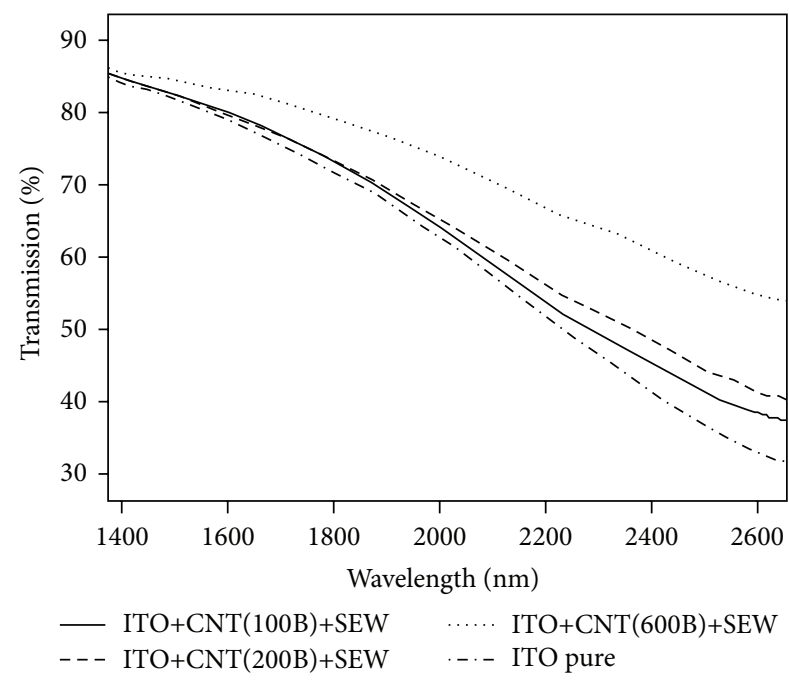

FIGURE 8: IR-transmittance spectra of the ITO-layers with laserdeposited CNTs and by additionally treated with the SEW.

Supérieure de Lyon, France); Professor I. Rau (University Politehnica of Bucharest, Romania), Dr. V. I. Studeonov, Dr. P. Ya. Vasilyev, Dr. S. V. Serov, and Ph.D. students A. A. Kukharchik and P. V. Kuzhakov (Vavilov State Optical Institute, St. Petersburg, Russia) for their help at the different steps and helpful discussion. The presented results are partially supported by Russian Foundation for Basic Research Fund, Grants no. 10-03-00916 (2010-2012) and no. 13-0300044 (2013-2015), and by the FP7 Program, Marie Curie International Researchers Exchange Proposal "BIOMOLEC" (2011-2015).

\section{References}

[1] D. Grasso and R. Gabriele-Campisi, "A DSC study of the liquid crystalline phases of salmon sperm DNA," Liquid Crystals, vol. 15, no. 5, pp. 701-708, 1993.
[2] I. C. Khoo, "Holographic grating formation in dye- and fullerene $\mathrm{C}_{60}$-doped nematic liquid-crystal film," Optics Letters, vol. 20, no. 20, pp. 2137-2139, 1995.

[3] H. Ono and N. Kawatsuki, "Orientational photorefractive gratings observed in polymer dispersed liquid crystals doped with fullerene," Japanese Journal of Applied Physics, vol. 36, no. 10, part 1, pp. 6444-6448, 1997.

[4] L. Lucchetti and F. Simoni, "Coarsening and phase separation in ultraviolet cured polymer dispersed liquid crystals," Journal of Applied Physics, vol. 88, no. 7, pp. 3934-3940, 2000.

[5] M. Even, B. Heinrich, D. Guillon, D. M. Guldi, M. Prato, and R. Deschenaux, "A mixed fullerene-ferrocene thermotropic liquid crystal: synthesis, liquid-crystalline properties, supramolecular organization and photoinduced electron transfer," Chemistry, vol. 7, no. 12, pp. 2595-2604, 2001.

[6] W. Lee and H.-C. Chen, "Diffraction efficiency of a holographic grating in a liquid-crystal cell composed of asymmetrically patterned electrodes," Nanotechnology, vol. 14, no. 9, pp. 987990, 2003.

[7] A.-H. Bae, T. Hatano, K. Sugiyasu, T. Kishida, M. Takeuchi, and S. Shinkai, "Supramolecular design of a porphyrin[60]fullerene photocurrent generation system on a DNA scaffold fabricated by a conjugate polymer film," Tetrahedron Letters, vol. 46, no. 18, pp. 3169-3173, 2005.

[8] I. C. Khoo, Y. Z. Williams, B. Lewis, and T. Mallouk, “+Photorefractive CdSe and gold nanowire-doped liquid crystals and polymer-dispersed-liquid-crystal photonic crystals," Molecular Crystals and Liquid Crystals, vol. 446, pp. 233-244, 2006.

[9] I. C. Khoo, A. Diaz, D. Kwon et al., "Nonlinear and electrooptics of nano-dispersed nematic liquid crystals with tunable negative-, zero-, and positive indices," Journal of Nonlinear Optical Physics and Materials, vol. 16, no. 3, pp. 381-399, 2007.

[10] X. Tong and Y. Zhao, "Liquid-crystal gel-dispersed quantum dots: reversible modulation of photoluminescence intensity using an electric field," Journal of the American Chemical Society, vol. 129, no. 20, pp. 6372-6373, 2007.

[11] I. A. Budagovsky, V. N. Ochkin, M. P. Smayev et al., "Interaction of light with a NLC-dendrimer system," Liquid Crystals, vol. 36, no. 1, pp. 101-107, 2009.

[12] E. Ouskova, J. Vapaavuori, and M. Kaivola, "Self-orienting liquid crystal doped with polymer-azo-dye complex," Optical Materials Express, vol. 1, no. 8, pp. 1463-1470, 2011.

[13] A. Firmino, J. G. Grote, F. Kajzar, J.-C. M’Peko, and A. Pawlicka, "DNA-based ionic conducting membranes," Journal of Applied Physics, vol. 110, no. 3, Article ID 033704, 2011.

[14] C. Blanc, D. Coursault, and E. Lacaze, "Ordering nano- and microparticles assemblies with liquid crystals," Liquid Crystals Reviews, vol. 1, no. 2, pp. 83-109, 2013.

[15] I. Dancus, V. I. Vlad, A. Petris et al., "Nonlinear optical properties of Rh610 sensitized DNA-CTMA characterized by $Z$-Scan," in ROMOPTO: Tenth Conference on Optics: Micro- to Nanophotonics III, vol. 8882 of Proceedings of SPIE, Bucharest, Romania, September 2012.

[16] Ch. Bosshard, K. Sutter, P. Günter, and G. Chapuis, "Linearand nonlinear-optical properties of 2-cyclooctylamino-5nitropyridine," Journal of the Optical Society of America, vol. B6, pp. 721-725, 1989.

[17] G. Lahajnar, I. Zupančič, R. Blinc, A. Zidanšek, R. Kind, and M. Ehrensperger, "NMR self-diffusion study of organic glasses: COANP, MBANP, PNP, NPP," Zeitschrift für Physik B Condensed Matter, vol. 95, no. 2, pp. 243-247, 1994. 
[18] J. Seliger, V. Zagar, R. Blinc, H. Arend, and P. Günter, "14N quadrupole coupling in COANP," Chemical Physics Letters, vol. 142, no. 5, pp. 334-335, 1987.

[19] A. Leyderman and Y. Cui, "Electro-optical characterization of a 2-cyclo-octylamino-5-nitropyridine thin organic crystal film," Optics Letters, vol. 23, no. 12, pp. 909-911, 1998.

[20] Y. Cui, J. Wu, N. Kamanina et al., "Dielectric study of dynamics of organic glasses," Journal of Physics D: Applied Physics, vol. 32, no. 24, pp. 3215-3221, 1999.

[21] N. Kamanina, A. Barrientos, A. Leyderman, Y. Cui, V. Vikhnin, and M. Vlasse, "Effect of fullerene doping on the absorption edge shift in COANP," Molecular Materials, vol. 13, no. 1-4, pp. 275-280, 2000.

[22] N. V. Kamanina, "Peculiarities of optical limiting effect in $\pi$-conjugated organic systems based on 2-cyclooctylamino-5nitropyridine doped with $\mathrm{C}_{70}$," Journal of Optics A: Pure and Applied Optics, vol. 3, no. 5, pp. 321-325, 2001.

[23] N. V. Kamanina, "Properties of optical limitation of radiation in $\pi$-conjugated organic systems doped with fullerenes (the COANP-C70 system)," Optics and Spectroscopy, vol. 90, no. 6, pp. 931-937, 2001.

[24] N. V. Kamanina, "Optical investigations of a $\mathrm{C}_{70}$-doped 2cyclooctylamino-5-nitropyridine-liquid crystal system," Journal of Optics A: Pure and Applied Optics, vol. 4, no. 5, pp. 571574, 2002.

[25] N. V. Kamanina and A. I. Plekhanov, "Mechanisms of optical limiting in fullerene-doped -conjugated organic structures demonstrated with polyimide and COANP molecules," Optics and Spectroscopy, vol. 93, no. 3, pp. 408-415, 2002.

[26] N. V. Kamanina and A. I. Plekhanov, "Optical limiting mechanisms in fullerene-containing Pi-conjugated organic materials: polyimide and COANP," in 7th International Symposium on Laser Metrology Applied to Science, Industry, and Everyday Life, vol. 4900 of Proceedings of SPIE, pp. 61-71, Novosibirsk, Russia, September 2002.

[27] N. V. Kamanina, A. V. Komolkin, and N. P. Yevlampieva, "Variation of the orientational order parameter in a nematic liquid crystal-COANP- $\mathrm{C}_{70}$ composite structure," Technical Physics Letters, vol. 31, no. 6, pp. 478-480, 2005.

[28] V. Likhomanova Svetlana and V. Kamanina Natalie, "Mechanisms of optical limiting in a COANP solution containing fullerenes $\mathrm{C}_{70}$ : applicability for the optoelectronics devices," Processing and Application of Ceramics, vol. 5, no. 4, pp. 229231, 2011.

[29] N. V. Kamanina and E. F. Sheka, "Optical limiters and diffraction elements based on a COANP-fullerene system: nonlinear optical properties and quantum-chemical simulation," Optics and Spectroscopy, vol. 96, no. 4, pp. 599-612, 2004.

[30] N. V. Kamanina, S. V. Serov, Y. Bretonniere, and C. Andraud, "Organic systems and their photorefractive properties under the nano- and biostructuration: scientific view and sustainable development," Journal of Nanomaterials, vol. 2015, Article ID 278902, 5 pages, 2015.

[31] I. C. Khoo, H. Li, and Y. Liang, "Observation of orientational photorefractive effects in nematic liquid crystals," Optics Letters, vol. 19, no. 21, pp. 1723-1725, 1994.

[32] N. V. Kamanina and D. P. Uskokovic, "Refractive index of organic systems doped with nano-objects," Materials and Manufacturing Processes, vol. 23, no. 6, pp. 552-556, 2008.

[33] N. V. Kamanina, P. V. Kuzhakov, S. V. Likhomanova, C. Andraud, I. Rau, and F. Kajzar, "Photorefractive, photoconductive, dynamic features and interfaces of the optical materials modified with nanoobjects," Nonlinear Optics Quantum Optics, vol. 45, no. 4, pp. 283-292, 2014.

[34] S. A. Akhmanov and S. Yu. Nikitin, Physical Optics, Moscow University Press, Moscow, Russia, 1998.

[35] S. A. Akhmanov and S. Yu. Nikitin, Physical Optics, Oxford University Press, New York, NY, USA, 1997.

[36] R. J. Collier, C. B. Burckhardt, and L. H. Lin, Optical Holography, Academic Press, New York, NY, USA, 1971.

[37] N. V. Kamanina and N. A. Vasilenko, "Influence of operating conditions and interface properties on dynamic characteristics of liquid-crystal spatial light modulators," Optical and Quantum Electronics, vol. 29, no. 1, pp. 1-9, 1997.

[38] N. V. Kamanina, A. Emandi, F. Kajzar, and A.-J. Attias, "Laserinduced change in the refractive index in the systems based on nanostructured polyimide: comparative study with other photosensitive structures," Molecular Crystals and Liquid Crystals, vol. 486, pp. 1-11, 2008.

[39] N. V. Kamanina, "Fullerene-dispersed nematic liquid crystal structures: dynamic characteristics and self-organization processes," Physics-Uspekhi, vol. 48, no. 4, pp. 419-427, 2005.

[40] N. V. Kamanina, "The effect of the charge transfer pathway during intermolecular complex formation on nonlinear optical and photoconducting properties of nanocomposites," Technical Physics Letters, vol. 38, no. 2, pp. 114-117, 2012.

[41] N. V. Kamanina, P. Y. Vasilev, V. I. Studentov, and Y. E. Usanov, "Strengthening transparent conductive coatings and 'soft' materials of the IR range when nanotechnologies are used," Journal of Optical Technology, vol. 75, no. 1, pp. 67-68, 2008.

[42] N. V. Kamanina and P. Ya. Vasilyev, "On the possibility of a homeotropic alignment in nematic liquid crystal elements using carbon nanotubes," Technical Physics Letters, vol. 35, no. 6, pp. 501-503, 2009.

[43] N. V. Kamanina, P. Y. Vasilyev, and V. I. Studeonov, "Features of nanostructured coatings obtained by laser deposition of carbon nanotubes," Technical Physics Letters, vol. 37, no. 2, pp. 106-108, 2011.

[44] N. V. Kamanina and V. N. Kidalov, "A study of the lining up of red blood cells in a nematic liquid crystal medium," Technical Physics Letters, vol. 22, no. 7, pp. 571-572, 1996.

[45] N. V. Kamanina, "Similarities and differences between the effects of orientation of red blood cells in a nematic liquidcrystal medium and the Fröhlich electrical vibrations," Technical Physics Letters, vol. 23, no. 12, pp. 902-905, 1997.

[46] A. A. Kamanin and N. V. Kamanina, "Erythrocyte induced structurization of a liquid crystal mesophase," Technical Physics Letters, vol. 32, no. 7, pp. 610-613, 2006.

[47] N. V. Kamanina, Y. A. Zubtcova, A. A. Kukharchik, C. Lazar, and I. Rau, "Control of the IR-spectral shift via modification of the surface relief between the liquid crystal matrixes doped with the lanthanide nanoparticles and the solid substrate," Optics Express, vol. 24, no. 2, pp. A270-A275, 2016. 

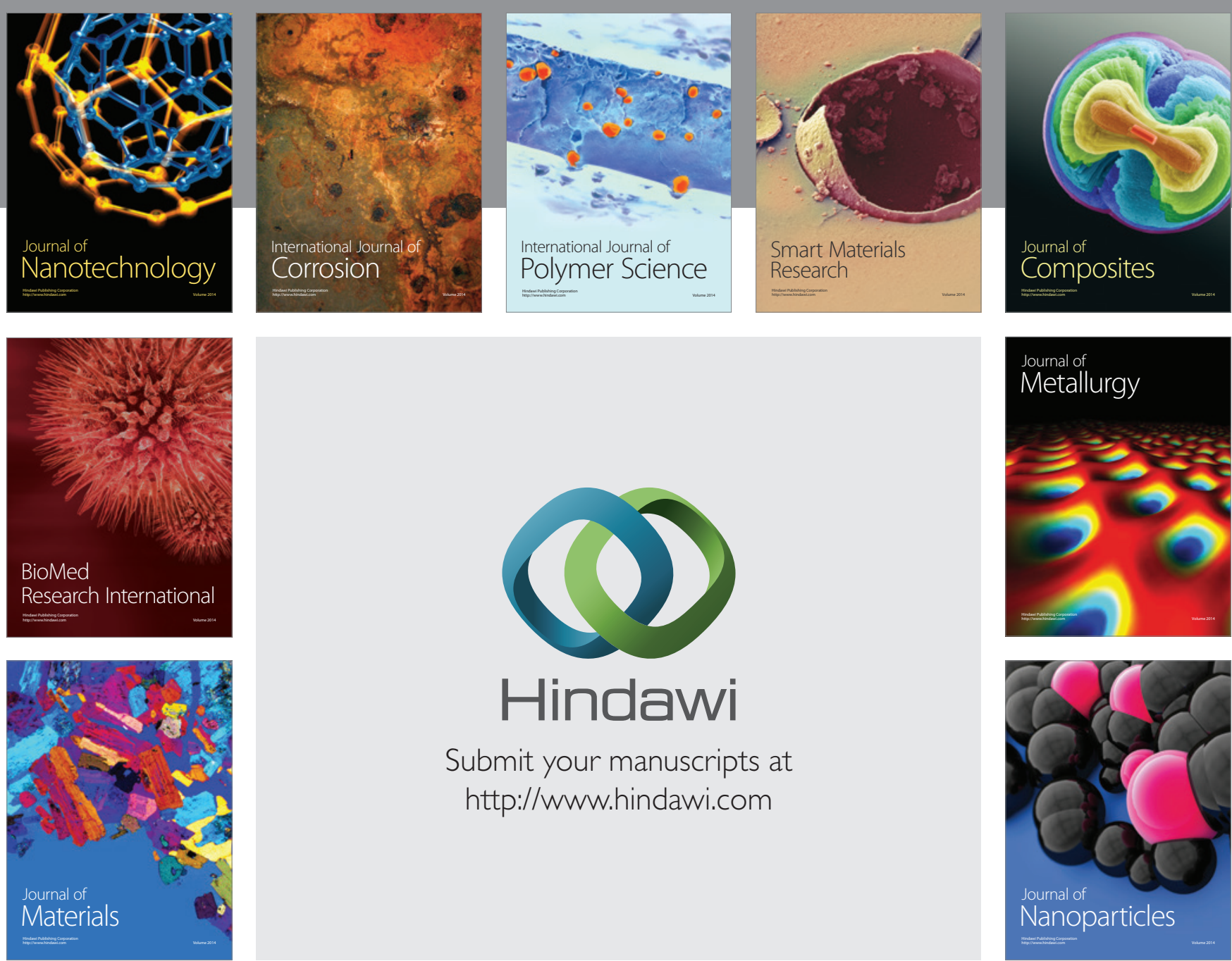

\section{Hindawi}

Submit your manuscripts at

http://www.hindawi.com

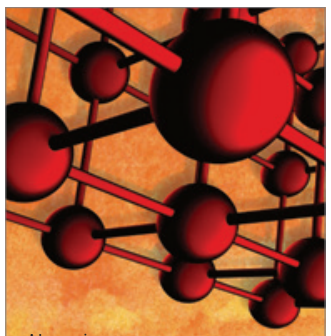

Materials Science and Engineering
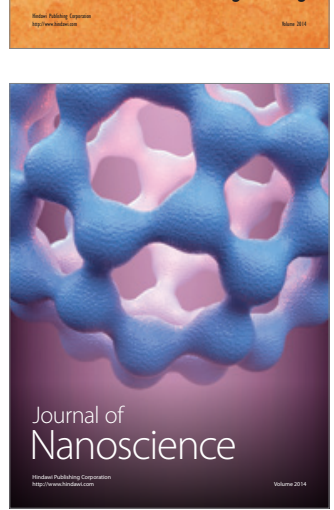
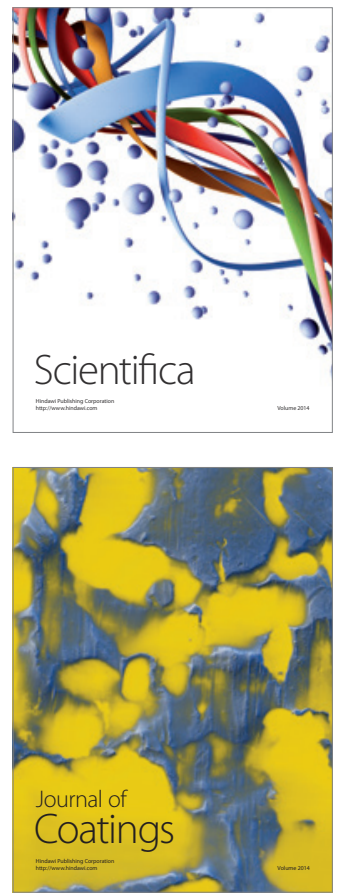
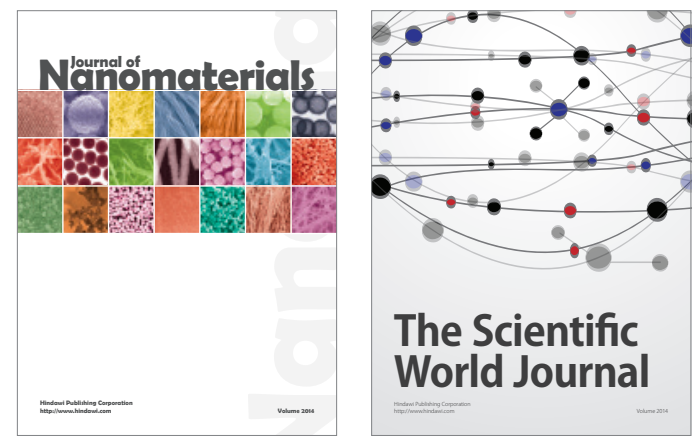

The Scientific World Journal
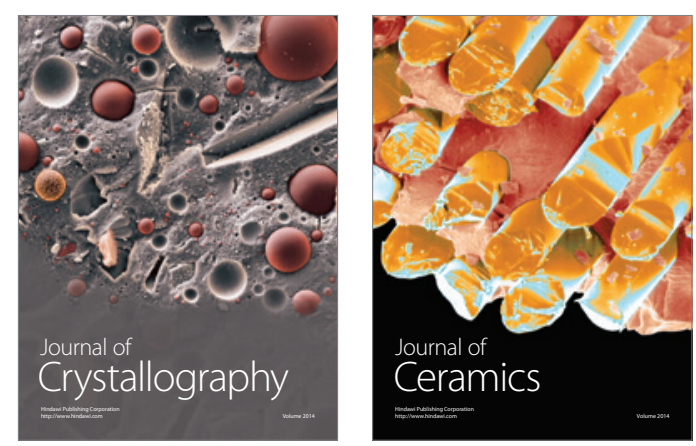
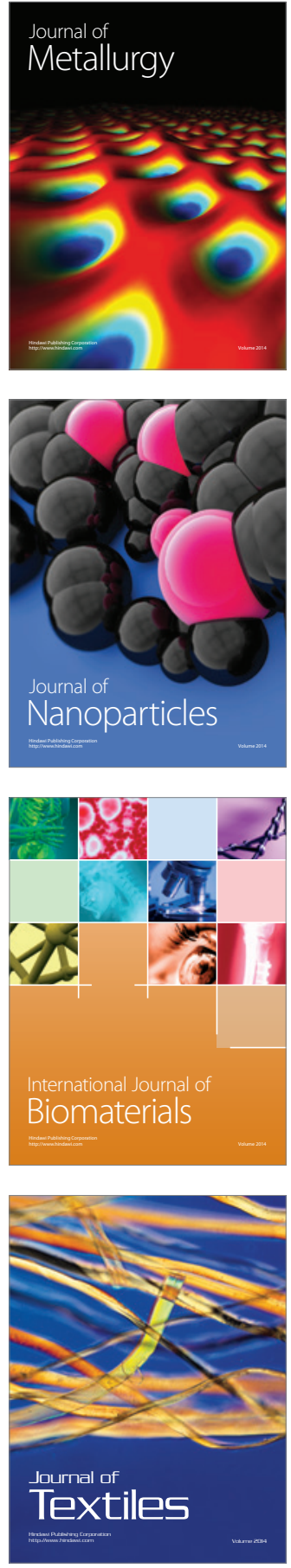